\title{
Producción de composta mediante descomposición aeróbica de residuos orgánicos en huertos de guayaba
}

Production of compost by aerobic decomposition of organic waste in guava orchards

\section{De Luna-Jiménez A. ${ }^{\circledR}$, Luna-Ruíz J.J. ${ }^{1}$, Martínez de Lara J. ${ }^{1}$}

${ }^{1}$ Departamento de Disciplinas Agrícolas, Centro de Ciencias Agropecuarias, Universidad Autónoma de Aguascalientes, km 3 de la carretera Jesús María-La Posta Zootécnica, apartado postal 2, Jesús María, CP: 20900, Tel. 9107400. Ext. 8115. Aguascalientes, México. Autor para

${ }^{凶}$ Autor para correspondencia: lunaji@yahoo.com

Recibido: $15 / 09 / 2018$

Aceptado: 15/11/2018

\section{RESUMEN}

La investigación se realizó en 2016, en Calvillo, Aguascalientes, con el propósito de elaborar abono orgánico a partir del compostaje de los residuos orgánicos producidos en las huertas de guayabo, mediante distintas proporciones de poda (ramas molidas), estiércol de bovino; fruta de desecho y zacate nativo, se elaboraron seis compostas. Los porcentajes más altos de $\mathrm{MO}, \mathrm{CO}$ y $\mathrm{N}$, se obtuvieron en la composta $4(\mathrm{P}(33.3 \%)+\mathrm{Z}(33.3 \%)+\mathrm{E}(33.3 \%))$. $\mathrm{La} \mathrm{CE} \mathrm{dSm}^{-1}$ y los porcentajes de $\mathrm{K}$ y $\mathrm{Mg}$ fueron mayores en composta $1(\mathrm{~F}(50 \%)+\mathrm{E}(50 \%))$. El porcentaje de Ca resulto elevado en la composta 5 (P $(50 \%)+\mathrm{E}(50 \%))$. El contenido más grande de $\mathrm{Zn} \mathrm{mg} \mathrm{kg}^{-1}$ y Mn mg kg-1 se registró en la composta 6 $(\mathrm{Z}(50 \%)+\mathrm{E}(50 \%))$. Se concluye que el compostaje produce un abono orgánico de calidad agronómica y puede utilizarse como mejorador físico-químico del suelo.

Palabras clave: Residuos orgánicos; compostaje; abono orgánico; guayaba.

\begin{abstract}
The research was carried out in 2016, in Calvillo, Aguascalientes, with the purpose of producing organic fertilizer from the composting of the organic waste produced in the guava orchards, by means of different proportions of pruning (ground branches), cow dung; waste fruit and native grass, six composts were made. The highest percentages of $\mathrm{MO}, \mathrm{CO}$ and $\mathrm{N}$ were obtained in compost 4 (P $(33.3 \%)+\mathrm{Z}(33.3 \%)+\mathrm{E}(33.3 \%))$. The $\mathrm{EC} \mathrm{dSm-1}$ and the percentages of $\mathrm{K}$ and $\mathrm{Mg}$ were higher in compost $1(\mathrm{~F}(50 \%)+\mathrm{E}(50 \%))$. The percentage of $\mathrm{Ca}$ resulted in high compost $5(\mathrm{P}(50 \%)+\mathrm{E}$ $(50 \%))$. The largest content of $\mathrm{Zn} \mathrm{mg} \mathrm{kg-1} \mathrm{and} \mathrm{Mn} \mathrm{mg} \mathrm{kg}-1$ was recorded in compost $6(\mathrm{Z}(50 \%)+\mathrm{E}$ $(50 \%))$. It is concluded that composting produces an organic fertilizer of agronomic quality and can be used as physical-chemical soil improver.
\end{abstract}

Keywords: Organic waste; composting; organic fertilizer; guava 


\section{INTRODUCCIÓN}

La investigación aborda el problema del manejo de los residuos orgánicos que se producen en las huertas de guayabo y se orienta a encontrar alternativas que disminuyan el impacto ecológico que provocan la descomposición de la fruta de desecho y la quema de ramas producto de la poda de los árboles y maleza que crece en el sitio, produciendo un abono que puede ser utilizado para mejorar las propiedades físico-químicas del suelo (Adak, Kumar, Singha, Shukla, \& Singh, 2014).

El compostaje es un proceso biológico aeróbico controlado, en el que intervienen numerosos microorganismos quienes destruyen la estructura molecular de los compuestos orgánicos, ocasiona cambios de temperatura y $\mathrm{pH}$ durante el proceso, liberando elementos nutritivos en formas asimilables para las plantas (Moran-Salazar, Marino-Marmolejo, Rodriguez-Campos, Davila-Vazquez, \& Contreras-Ramos, 2016). Asimismo se produce materia orgánica estable, libre de patógenos y disponible para ser utilizada como mejorador de suelos (Antunes, Scoriza, da Silva, \& Correia, 2016).

Probablemente halla poco por descubrir sobre los fundamentos biológicos del proceso y la interacción con las condiciones fisicoquímicas del suelo, pero queda mucho por investigar respecto al efecto en el rendimiento y beneficios del uso de abonos orgánicos en el suelo, además de aprender la manera correcta de reciclar los residuos (Islam, Ferdous, Akter, Hossain, \& Nandwani, 2017).

El reciclaje puede reducir el efecto contaminante que estos residuos causan al ambiente y permitir su reutilización como insumos en la agricultura. La adición al suelo de materiales biodegradables conlleva a la síntesis de complejos orgánicos que unen las partículas minerales en agregados, se incrementa el espacio poroso, mejora la infiltración y el almacenamiento del agua (Ankenbauer \& Loheide, 2017).

Los compuestos orgánicos constituyen además, fuente de nutrientes esenciales. Se ha estimado que la materia orgánica contiene más del $50 \%$ del carbono que sirve como fuente de energía para la población microbiana encargada de descomponer los residuos (Ram,

Singha, \& Bhriguvanshi, 2014).

El compostaje es una técnica muy antigua que consiste en mezclar desechos animales y vegetales que al proporcionarles la humedad, aireación y temperatura favorables, se propicia que los microorganismos conviertan estos materiales en compuestos orgánicos estabilizados (Ribeiro, Souza, Costa, de Castro, \& Dias, 2017).

La composta madura, producto final del proceso de descomposición, es un abono orgánico altamente humificado. $\mathrm{La}$ altas temperaturas $\left(45-60^{\circ} \mathrm{C}\right)$, durante la fase termofílica, causan la muerte de patógenos y semillas de malezas evitando que dañen a los cultivos (de Souza, Rozane, de Amorim, Modesto, \& Natale, 2014).

El estudio del compostaje de residuos orgánicos mezclados, reviste particular interés en Calvillo, Aguascalientes por el elevado costo de los fertilizantes de alta solubilidad y la necesidad de disponer de abono orgánico para utilizarlo en el abonado del guayabo. Por lo expuesto, se planteó el objetivo de aprovechar la disponibilidad de los residuos orgánicos, para elaborar composta de calidad agronómica. 


\section{MATERIALES Y MÉTODOS}

La investigación se realizó durante 2016, en San Tadeo, perteneciente al municipio de Calvillo, Aguascalientes. Los materiales utilizados fueron: ramas provenientes de la poda de los árboles, estiércol de bovino; fruta de desecho y zacate cosechado en las huertas del lugar (Cuadro 1).

Cuadro 1. Composición química de los materiales usados en la composta

\begin{tabular}{ccccc}
\hline Análisis & Poda & Estiércol & Fruto & Zacate \\
\hline $\mathrm{C}: \mathrm{N}$ & $80: 1$ & $25: 1$ & $30: 1$ & $60: 1$ \\
$\mathrm{~N} \%$ & 1.60 & 1.00 & 0.98 & \\
$\mathrm{P} \%$ & 0.18 & 0.18 & 0.21 & 0.22 \\
$\mathrm{~K} \%$ & 1.56 & 0.54 & 0.87 & \\
$\mathrm{Ca} \%$ & 1.37 & 2.72 & 0.48 & 1.0 \\
$\mathrm{Mg} \%$ & 0.33 & 0.65 & 0.12 & \\
$\mathrm{Na} \%$ & 0.21 & & 0.13 & \\
\hline
\end{tabular}

$\mathrm{C}: \mathrm{N}=$ Relación carbono nitrógeno; $\mathrm{N}=$ =nitrógeno; $\mathrm{P}=$ fósforo; $\mathrm{K}=$ potásio; $\mathrm{Ca}=$ cálcio; $\mathrm{Mg}=$ =magnésio y $\mathrm{Na}=$ sódio

\section{Recolección y acondicionamiento de los materiales}

Como resultado de la poda de los árboles quedan dispersas en la huerta una gran cantidad de ramas, se procedió a su recolección haciendo montones y a continuación, se acarrearon al lugar donde se molieron, utilizando un molino de martillos, con el cual se logró una molienda de partícula fina. Se llenaron costales y se transportaron al lugar donde se construyeron las pilas. Del mismo modo el zacate se cosecho en la huerta, se dejó secar al sol y se molió. Así mismo el estiércol se obtuvo en los corrales de la localidad y las guayabas de desecho, se consiguieron en un centro de acopio donde se selecciona y empaca la fruta (Figura 1).

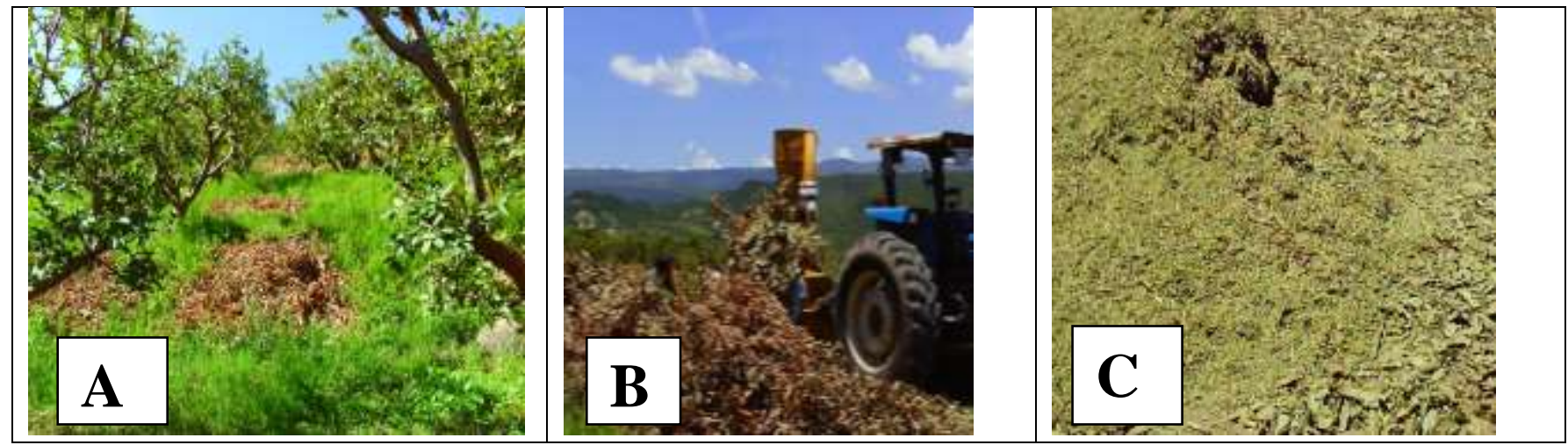



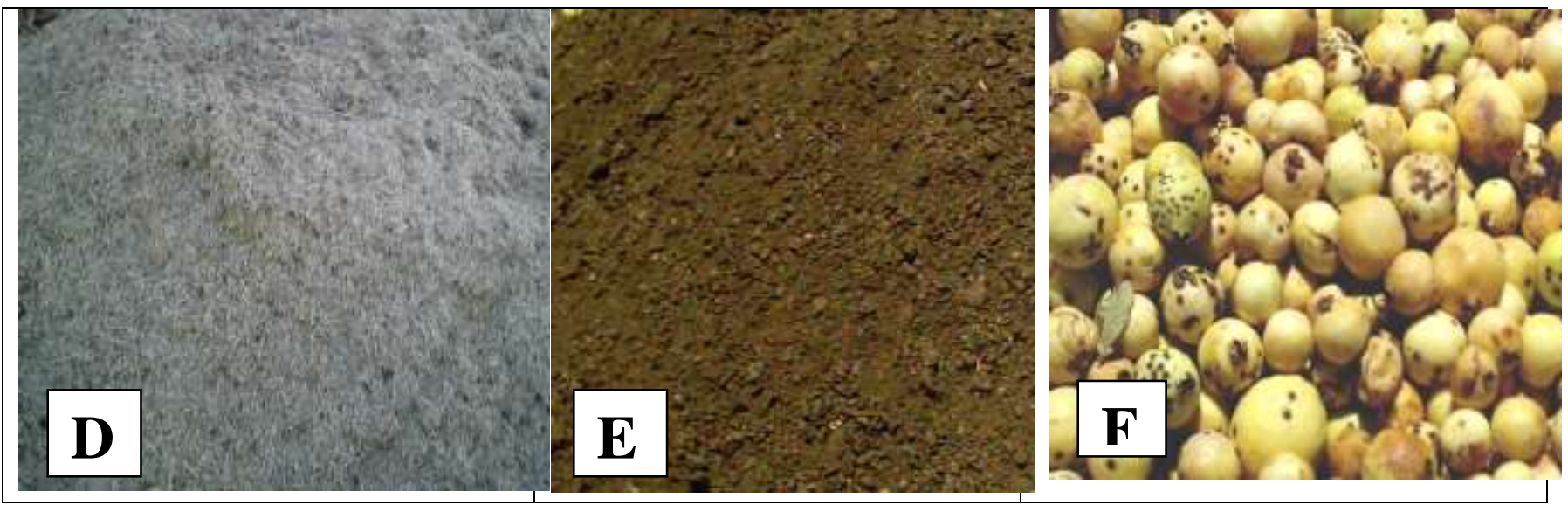

Figura 1. Materiales utilizados en las compostas: $\mathbf{A}=$ Poda de árboles, $\mathbf{B}=$ Molienda de Ramas, $\mathbf{C}=$ Ramas molidas, $\mathbf{D}=$ Zacate molido, $\mathbf{E}=$ Estiércol de bovino, $\mathbf{F}=$ Fruta de desecho. Elaboración propia.

Definición y Descripción de tratamientosCon los materiales indicados en la tabla 1 , se prepararon seis compostas por triplicado, cada tratamiento fue una composta (Cuadro 2).

Cuadro 2. Tratamientos y su descripción

\begin{tabular}{cll}
\hline Tratamiento & Proporción en la Mezcla & Descripción \\
\hline C1 & $\mathrm{F}(50 \%)+\mathrm{E}(50 \%)$ & Fruta + Estiércol \\
C2 & $\mathrm{F}(33.3 \%)+\mathrm{P}(33.3 \%)+\mathrm{E}(33.3 \%)$ & Fruta + Poda + Estiércol \\
C3 & $\mathrm{F}(25 \%)+\mathrm{P}(25 \%)+\mathrm{Z}(25 \%)+\mathrm{E}(25 \%)$ & Fruta + Poda + Zacate + Estiércol \\
C4 & $\mathrm{P}(33.3 \%)+\mathrm{Z}(33.3 \%)+\mathrm{E}(33.3 \%)$ & Poda + Zacate + Estiércol \\
C5 & $\mathrm{P}(50 \%)+\mathrm{E}(50 \%)$ & Poda + Estiércol \\
C6 & $\mathrm{Z}(50 \%)+\mathrm{E}(50 \%)$ & Zacate + Estiércol \\
\hline
\end{tabular}

$\mathrm{C}=$ Composta; $\mathrm{F}=$ Fruta; $\mathrm{E}=$ Estiércol; $\mathrm{P}=$ Poda $\mathrm{y} \mathrm{Z}=\mathrm{Zacate}$.

\section{Construcción de pilas}

Se construyeron las mezclas según el método de la pila (Antunes et al., 2016). Los materiales se colocaron en capas sobrepuestas de acuerdo con las proporciones indicadas en la tabla 2, utilizando primero los materiales con partícula más grande procurando aplicar el estiércol en la parte superior de cada montón, que alcanzó una altura aproximada de 1 metro y una base de 2 metros. Enseguida se humedecieron las pilas, se estimó la humedad tomando un puño de la mezcla, se presionó con la mano y cuando salieron dos o tres gotas de agua, se estimó que la humedad se encontraba en un $60 \%$ considerado adecuado, se continuó humedeciendo periódicamente las pilas para mantener la humedad en $45-55 \%$ considerado favorable a la actividad microbiana, se cubrieron con plástico negro para minimizar la pérdida de calor y mantener la humedad.

Después de la fase termofílica o de máxima temperatura se voltearon los materiales hasta hacer una nueva pila, con una periodicidad semanal, esta labor permitió mezclar aquellas capas externas, frías, no descompuestas con el núcleo central más biodegradado, reiniciándose el proceso de transformación microbiológica (Figura 2). 


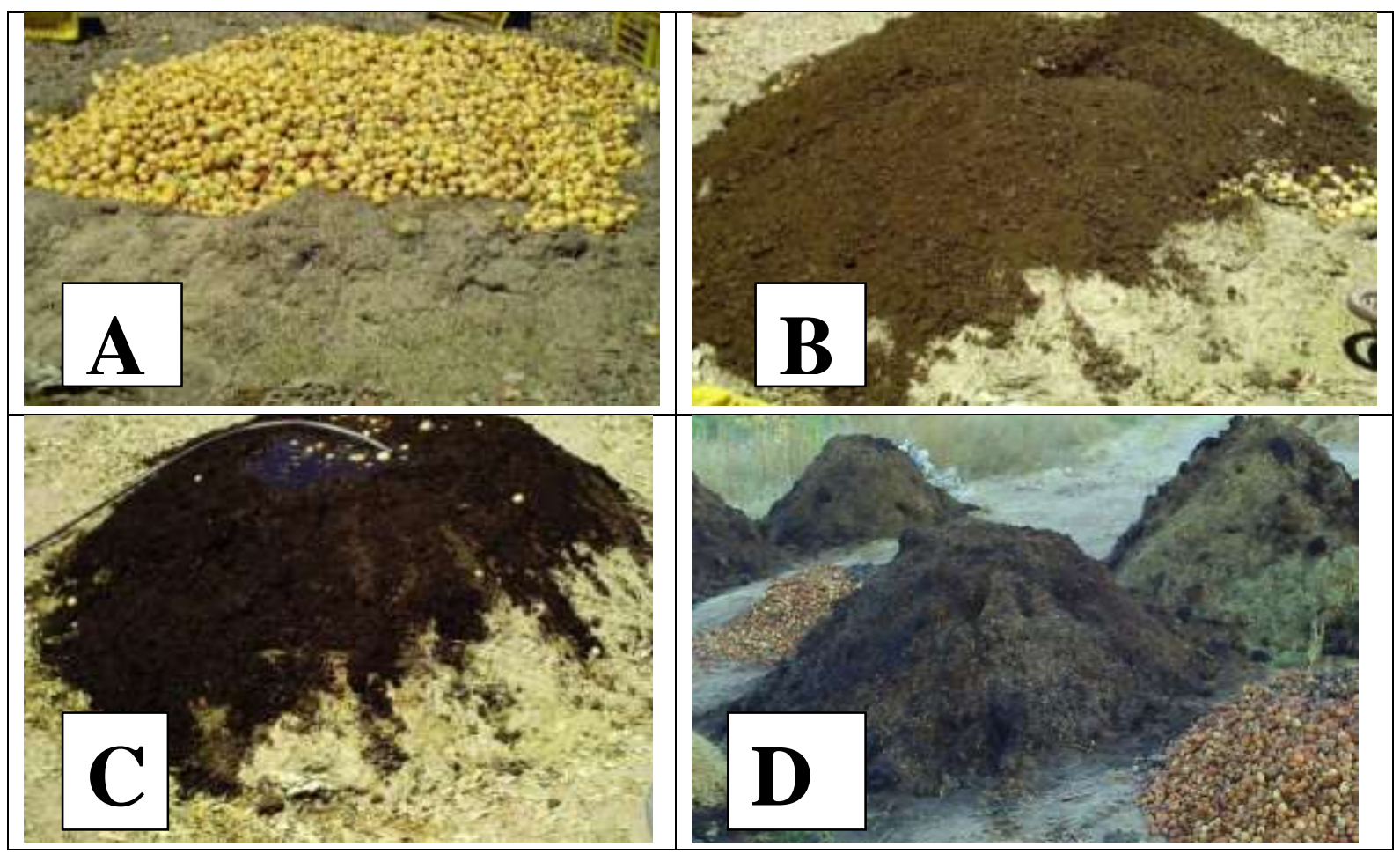

Figura 2 Construcción de pilas de composteo: $\mathbf{A}=$ Muestra en la parte superior las guayabas de desecho, $\mathbf{B}=\mathrm{El}$ estiércol sobre las guayabas, $\mathbf{C}=$ Aplicación de agua para humedecer las pilas $\mathrm{y}$ $\mathbf{D}=$ Señala un estado avanzado de descomposición de los materiales. Elaboración propia.

\section{Medición de variables}

Cuadro 3. La metodología correspondiente a cada variable, se menciona a continuación:

\begin{tabular}{cccl}
\hline Variable & Unidades & Metodología utilizada \\
\hline $\mathrm{T}$ & ${ }^{\circ} \mathrm{C}$ & Termómetro (vástago $50 \mathrm{~cm}$. ) \\
$\mathrm{pH}$ & $\mathrm{pH}$ & Potenciómetro relación 1:2 p/v (peso/agua) \\
$\mathrm{MO}$ & $\%$ & Walkley y Black \\
$\mathrm{CO}$ & $\%$ & Walkley y Black \\
$\mathrm{CE}$ & $\mathrm{dSm}^{-1}$ & Puente de conductividad en extracto de pasta \\
$\mathrm{N}$ & $\%$ & Destilación Semimicro-kjeldahl \\
$\mathrm{P}$ & $\%$ & Fotocolorimetría: Red. Molibdo-vanadato \\
$\mathrm{K}$ & $\%$ & Absorción Atómica \\
$\mathrm{Ca}$ & $\%$ & Absorción Atómica \\
$\mathrm{Mg}$ & $\%$ & Absorción Atómica \\
$\mathrm{Fe}$ & $\mathrm{mg} \mathrm{kg}-1$ & Absorción Atómica \\
$\mathrm{Cu}$ & $\mathrm{mg} \mathrm{kg}^{-1}$ & Absorción Atómica \\
$\mathrm{Zn}$ & $\mathrm{mg} \mathrm{kg}^{-1}$ & Absorción Atómica \\
$\mathrm{Mn}$ & $\mathrm{mg} \mathrm{kg}^{-1}$ & Absorción Atómica \\
$\mathrm{B}$ & $\mathrm{mg} \mathrm{kg}^{-1}$ & Foto colorimetría de Azometina-H
\end{tabular}

$\mathrm{T}=$ Temperatura, $\mathrm{pH}=$ Potencial hidrógeno, $\mathrm{MO}=$ Materia orgánica, $\mathrm{CO}=$ Carbono orgánico, $\mathrm{CE}=$ Conductividad eléctrica, $\mathrm{N}=$ Nitrógeno, $\mathrm{P}=$ Fósforo, $\mathrm{K}=$ Potasio, $\mathrm{Ca}=\mathrm{Calcio}, \mathrm{Mg}=\mathrm{Magnesio}$, $\mathrm{Fe}=$ Fierro, $\mathrm{Cu}=$ Cobre, $\mathrm{Zn}=\mathrm{Zinc}, \mathrm{Mn}=$ Manganeso, $\mathrm{B}=$ Boro. 


\section{RESULTADOS Y DISCUSIÓN}

\section{Temperatura}

Avanzando en el tiempo de composteo se encontraron diferencias significativas $(\mathrm{p}<0.001)$ en la temperatura registrada dentro de las pilas de composta, lo que se explica por la proporción de los componentes que influyen en la actividad de los microorganismos que realizan el proceso de descomposición microbiológica. La C1 (F (50\%) + E (50\%)), registró la temperatura más alta, en promedio $62.2^{\circ} \mathrm{C}$. Este resultado concuerda con lo reportado por (Valdrighi et al., 1996), quienes afirman que para una adecuada esterilización de la composta se requieren periodos entre 4 y 15 días con temperaturas superiores a $55^{\circ} \mathrm{C}$ o una hora por encima de $70^{\circ} \mathrm{C}$ (Ribeiro et al., 2017). La C6 (Z (50\%) + E (50\%)), alcanzó temperaturas bajas del orden de $48^{\circ} \mathrm{C}$, lo que pudo deberse en parte a que el zacate es altamente lignificado, alto en carbono y pobre en nitrógeno lo que dificulta su descomposición, también el exceso de humedad ocasionó la formación de grumos ocasionando deficiente oxigenación y dificultad para destruir estos agregados con los volteos. Esta situación reafirma la necesidad de incorporar un material de soporte que aporte estructura y mejore la aireación de la pila durante el proceso. Las compostas: $\mathrm{C} 1(\mathrm{~F}(50 \%)+\mathrm{E}(50 \%)), \mathrm{C} 2(\mathrm{~F}$ $(33.3 \%)+\mathrm{P}(33.3 \%)+\mathrm{E}(33.3 \%))$ y $\mathrm{C} 3(\mathrm{~F}$ $(25 \%)+\mathrm{P}(25 \%)+\mathrm{Z}(25 \%)+\mathrm{E}(25 \%))$, registraron alta temperatura debido a la combinación de fruta y estiércol que son los materiales más ricos en nitrógeno y por ello los microorganismos dispusieron de más alimento lo que permitió su multiplicación y mayor eficiencia en su actividad de degradación. Las compostas: $\mathrm{C} 4(\mathrm{P}(33.3 \%)+\mathrm{Z}(33.3 \%)+\mathrm{E}$ $(33.3 \%)), \mathrm{C} 5(\mathrm{P}(50 \%)+\mathrm{E}(50 \%))$ y $\mathrm{C} 6(\mathrm{Z}$ $(50 \%)+\mathrm{E}(50 \%))$, fueron las de menor temperatura promedio ya que el componente nitrogenado únicamente fue el estiércol.

Cuadro 4. Análisis de Varianza y prueba de comparación de medias

\begin{tabular}{|c|c|c|c|c|c|c|c|c|c|}
\hline Tratamiento & MO* & $\mathrm{CO}^{*}$ & $\mathrm{CE}^{* *}$ & $\mathrm{~N}^{*}$ & $\mathrm{~K}^{* *}$ & $\mathrm{Ca}^{*}$ & Mg* & $\mathrm{Zn} *$ & Mn* \\
\hline $\mathrm{C} 1$ & $22.8 \mathrm{~b}$ & $13.3 b$ & $13.2 \mathrm{a}$ & $0.8 \mathrm{ab}$ & $2.9 \mathrm{a}$ & $1.0 \mathrm{~b}$ & $0.82 \mathrm{a}$ & $151 \mathrm{a}$ & $371 \mathrm{a}$ \\
\hline $\mathrm{C} 2$ & $27.8 \mathrm{ab}$ & 16.1ab & $6.1 \mathrm{~b}$ & $0.8 \mathrm{ab}$ & $1.4 \mathrm{~b}$ & $1.3 \mathrm{ab}$ & $0.80 \mathrm{ab}$ & $123 \mathrm{ab}$ & $352 \mathrm{a}$ \\
\hline C3 & 28.0ab & $16.5 b$ & $7.0 \mathrm{~b}$ & $0.9 \mathrm{ab}$ & $1.5 \mathrm{ab}$ & $1.1 \mathrm{~b}$ & $0.70 \mathrm{ab}$ & $127 a b$ & $333 a$ \\
\hline $\mathrm{C} 4$ & $46.2 \mathrm{a}$ & $26.8 \mathrm{a}$ & $5.1 \mathrm{~b}$ & $1.1^{\mathrm{a}}$ & $1.2 \mathrm{~b}$ & $1.5 \mathrm{ab}$ & $0.58 b$ & $85 b$ & $233 a$ \\
\hline C5 & $33.6 a b$ & $19.5 \mathrm{ab}$ & $5.8 \mathrm{~b}$ & $0.9 \mathrm{ab}$ & $1.2 \mathrm{~b}$ & $1.9 \mathrm{a}$ & $0.79 a$ & $125 \mathrm{ab}$ & $339 a$ \\
\hline C6 & $22.7 \mathrm{~b}$ & $13.7 \mathrm{~b}$ & 8.6ab & $0.7 b$ & $1.3 b$ & $1.1 \mathrm{~b}$ & $0.80 \mathrm{a}$ & $159 a$ & $393 a$ \\
\hline $\begin{array}{l}\mathrm{MO}=\text { Materia } \\
\mathrm{N}=\text { Nitrógeno } \\
\text { Mn=Mangane } \\
\text { Promedios co }\end{array}$ & rgánica & 6), $\mathrm{CC}$ & & & & $\mathrm{E}=$ Conc & tividad & $\begin{array}{l}\text { léctric } \\
\text { Zinc }\end{array}$ & $\begin{array}{r}\left.\mathrm{dSm}^{-1}\right), \\
\left.\mathrm{kg}^{-1}\right), \\
(0.01) .\end{array}$ \\
\hline
\end{tabular}


En la cuadro 4 se presentan los resultados y se aprecia que los porcentajes de $\mathrm{MO}, \mathrm{CO}$ y $\mathrm{N}$, fueron mayores en la $\mathrm{C} 4(\mathrm{P}(33.3 \%)+\mathrm{Z}$ $(33.3 \%)+\mathrm{E}(33.3 \%))$ y menor en la $\mathrm{C} 1(\mathrm{~F}$ $(50 \%)+\mathrm{E}(50 \%))$ у C6 (Z (50\%) + E (50\%)), Resultado que se explica por el componente poda de ramas como fuente de carbono. La CE $\mathrm{dSm}^{-1}$ en $\mathrm{C} 1(\mathrm{~F}(50 \%)+\mathrm{E}(50 \%))$, fué superior debido a la alta proporción de fruta y estiércol, este resultado señala que el estiércol tiene el inconveniente de aportar alto contenido de sales. En K se observa que la C1 (F (50\%) + E $(50 \%)$ ), resultó superior a las demás, este resultado es dado que la proporción de fruta y estiércol es alta.

En Ca la C5 (P (50\%) + E (50\%)), resulto superior debido al componente poda.

En Mg la C1 (F (50\%) + E (50\%)), fue superior a las demás, atribuible al contenido de fruta. La C6 $(\mathrm{Z}(50 \%)+\mathrm{E}(50 \%))$, mostró un mayor contenido de $\mathrm{Zn}$ y $\mathrm{Mn}$ con respecto a la $\mathrm{C} 4$ (P $(33.3 \%)+Z(33.3 \%)+E(33.3 \%))$ a causa de que las cantidades de los componentes de la mezcla son altos.

\section{CONCLUSIONES}

La investigación señala la factibilidad técnica de convertir los residuos orgánicos que son fuente inicial de contaminación, mediante su biotransformación en un abono orgánico que se puede utilizar como mejorador de suelo. Los resultados señalan que los residuos orgánicos deben utilizarse en proporción balanceada para obtener composta de buena calidad. La alta relación $\mathrm{C} / \mathrm{N}$ en el zacate y en la poda, puede dificultar la actividad microbiana y causar por compactación una deficiencia de oxigenación, por lo que deberá realizarse volteos frecuentes, al menos uno por semana y mantener la humedad cerca del punto de saturación. La C4, con proporciones iguales de poda, zacate $\mathrm{y}$ estiércol, aportó la mayor cantidad de MO, CO y N. En C1, la elevada CE, el aporte de $\mathrm{Ca}, \mathrm{Mg}$ y el alto contenido de $\mathrm{Zn}$ es atribuible a las proporciones utilizadas de fruta y estiércol.

\section{LITERATURA CITADA}

Adak, T., Kumar, K., Singha, A., Shukla, S. K., \& Singh, V. K. (2014). ASSESSING SOIL CHARACTERISTICS AND GUAVA ORCHARD PRODUCTIVITY AS INFLUENCED BY ORGANIC AND INORGANIC SUBSTRATES. Journal of Animal and Plant Sciences, 24(4), 11571165.

Ankenbauer, K. J., \& Loheide, S. P. (2017). The effects of soil organic matter on soil water retention and plant water use in a meadow of the Sierra Nevada, CA. Hydrological Processes, 31(4), 891-901. https://doi.org/10.1002/hyp.11070

Antunes, L. F. D., Scoriza, R. N., da Silva, D. G., \& Correia, M. E. F. (2016). Production and efficiency of organic compost generated by millipede activity. Ciencia Rural, 46(5), 815-819. https://doi.org/10.1590/0103-8478cr20150 de Souza, H. A., Rozane, D. E., de Amorim, D. A., Modesto, V. C., \& Natale, W. (2014). AGRONOMIC USE OF BY-PRODUCT OF GUAVA PROCESSING INDUSTRY - I - CHEMICAL SOIL PROPERTIES. Revista Brasileira De Fruticultura, 36(3), 713-724. https://doi.org/10.1590/0100-294 Islam, M. A., Ferdous, G., Akter, A., Hossain, M. M., \& Nandwani, D. (2017). Effect of Organic, Inorganic Fertilizers and Plant Spacing on the Growth and Yield of Cabbage. Agriculture-Basel, 7(4). https://doi.org/10.3390/agriculture704003 Moran-Salazar, R. G., Marino-Marmolejo, E. N., Rodriguez-Campos, J., Davila- 
Vazquez, G., \& Contreras-Ramos, S. M. (2016). Use of agave bagasse for production of an organic fertilizer by pretreatment with Bjerkandera adusta and vermicomposting with Eisenia fetida. Environmental Technology, 37(10), 1220-

1231.https://doi.org/10.1080/09593330.20 15.1108368

Ram, R. A., Singha, A., \& Bhriguvanshi, S. R. (2014). Response of on farm produced organic inputs on soil, plant nutrient status, yield and quality of guava (Psidium guajava) cv Allahabad Safeda. Indian Journal of Agricultural Sciences, 84(8), 962-967
Ribeiro, N. D., Souza, T. P., Costa, L., de Castro, C. P., \& Dias, E. S. (2017). Microbial additives in the composting process. Ciencia E Agrotecnologia, 41(2), 159-168 https//doi.org/10.1590/141370542017412038216

Valdrighi, M. M., Pera, A., Agnolucci, M., Frassinetti, S., Lunardi, D., \& Vallini, G. (1996). Effects of compost-derived humic acids on vegetable biomass production and microbial growth within a plant (Cichorium intybus)-soil system: A comparative study. Agriculture Ecosystems \& Environment, 58(2-3), 133144.https://doi.org/10.1016/0167-8809(96) 01031-6

Copyright (c) 2018 Alfonso De Luna Jim énez, José de Jesús Luna Ruiz y J. Martinez de Lara

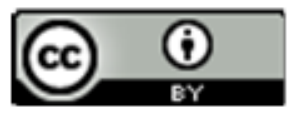

Este tex to está protegido por una licencia CreativeCommons $\underline{4.0}$

Usted es libre para Compartir — copiar y redistribuir el material en cualquier medio o form ato-y Adaptar el documento — remezclar, transformar y crear a partir del material- para cualquier propósito, incluso para fi nes comerciales, siempre que cumpla la condición de:

Atribución: Usted debe dar crédito a la obra original de manera adecuada, proporcionar un enlace a la licencia, e in dicar si se han realizado cam bios. Puede hacerlo en cualquier form a razonable, pero no de form a tal que sugiera que tiene el apoyo del licenciante o lo recibe por el uso que hace de la obra.

Resumendelicencia - Textocompletodelalicencia 